\section{Low thyroid function}

Abbreviations: TSH, thyroid stimulating hormone; AACE, American association of clinical endocrinologists; TRH, thyroid releasing hormone; BBT, basal body temperature; MS, multiple sclerosis; $\mathrm{mIU} / \mathrm{L}$, milli-international units per liter; $\mathrm{ng} / \mathrm{dl}$, nanograms per deciliter; pg/dl, picograms per deciliter

\section{Editorial}

The thyroid, a butterfly-shaped gland in the neck, can have a dramatic impact on a huge variety of bodily functions, and if you're a woman over 35 your odds of a thyroid disorder are high more than $30 \%$ by some estimates. At least 30 million Americans have a thyroid disorder and half 15 million are silent sufferers who are un-diagnosed, according to The American Association of Clinical Endocrinologists. Women are as much as 10times as likely as men to have a thyroid problem. Feeling tired and having no energy are issues associated with lots of conditions, but they are strongly linked with hypothyroidism, the disorder that is the result of too little thyroid hormone. If you are still tired in the morning or all day after a full night's sleep that is a clue that your thyroid may be underactive. Too little thyroid hormone coursing through your bloodstream and cells means your muscles aren't getting that get-going signal. Fatigue is the number one symptom I see, says Dr. Miller. It is the kind of fatigue where you're still tired in the morning after a full night sleep that is a clue that you're not simply sleep deprived; your thyroid may be underactive.

The classic signs of a sluggish thyroid gland include weight gain, lethargy, poor quality hair and nails, hair loss, dry skin, fatigue, cold hands and feet, and constipation and these symptoms are relatively well known.

However, some of the conditions you might not associate with your thyroid include:
i. High cholesterol
ii. Irregular menstruation
iii. Low libido
iv. Infertility
v. Gum disease
vi. Fluid retention

vii. Skin conditions such as acne and eczema

viii. Memory problems

\section{ix. Poor stamina}

And there are in fact, many more conditions that can be associated with poor thyroid function. Your thyroid plays a part in nearly every physiological process. When it is out of balance, so are you. This is why it is so important to understand how your thyroid gland works and what can cause it to run amok. The sad fact is, half of all people with hypothyroidism are never diagnosed. And of those who are diagnosed, many are inadequately treated, resulting in partial recovery at best.

\section{Hypothyroidism:The hidden epidemic}

Hypothyroidism simply means you have a sluggish or underactive
Volume 2 Issue 4 - 2015

\author{
George Grant \\ World organization of Natural Medicine, Canada
}

Correspondence: George Grant, World organization of Natural Medicine, Richmond Hill, Ontario, Canada, Tel 4I6 562 3140, Email drgrantwellness@gmail.com

Received: July 21, 2015 | Published: August 03, 2015

thyroid, which is producing less than adequate amounts of thyroid hormone. Subclinical hypothyroidism means you have no obvious symptoms and only slightly abnormal lab tests. I will be discussing these tests much more as we go on since they are a source of great confusion for patients, as well as for many health practitioners. Thyroid problems have unfortunately become quite common. The same lifestyle factors contributing to high rates of obesity, cancer, and diabetes are wreaking havoc on your thyroid sugar processed foods, stress, environmental toxins, and lack of exercise are heavy contributors. More than 10percent of the general population in the United States and 20percent of women over the age of 60 has subclinical hypothyroidism. But only a small percentage of these people are being treated.

\section{Why is that?}

Much of it has to do with misinterpretation and misunderstanding of lab tests, particularly TSH (thyroid stimulating hormone). Most physicians believe that if your TSH value is within the range of normal, your thyroid is fine. But more and more physicians are discovering that the TSH value is grossly unreliable for diagnosing hypothyroidism. And the TSH range for normal keeps changing! In an effort to improve diagnosis of thyroid disease, in 2003 the American Association of Clinical Endocrinologists (AACE) revised the normal TSH range as 0.3 to 3.042 . The previous range was defined as 0.5 and 5.0, which red-flagged only the most glaring hypothyroidism cases. However, the new range is still not wholly reliable as the sole indicator of a sulky thyroid gland. You simply cannot identify one $\mathrm{TSH}$ value that is normal for every person, regardless of age, health, or other factors. Having said that, though, most physicians who carefully follow this condition recognize that any TSH value greater than 1.5 could be a strong indication that an underactive thyroid is present. Your TSH value is only part of the story, and your symptoms, physical findings, genetics, lifestyle, and health history are also important considerations. Only when physicians learn to treat the patient and not the lab test will they begin to make headway against thyroid disease. Understanding How Your Thyroid Works Is Step One.

The thyroid gland is in the front of your neck and is part of your endocrine, or hormonal, system. It produces the master metabolism hormones that control every function in your body. 3 Thyroid hormones interact with all your other hormones including insulin, cortisol, and sex hormones like estrogen, progesterone, and testosterone. The fact that these hormones are all tied together and 
in constant communication explains why an unhappy thyroid is associated with so many widespread symptoms and diseases. This small gland produces two major thyroid hormones: T4 and T3. About 90percent of the hormone produced by the gland is in the form of $\mathrm{T} 4$, the inactive form. Your liver converts this T4 into T3, the active form, with the help of an enzyme. Your thyroid also produces T2, yet another hormone, which currently is the least understood component of thyroid function and the subject of much ongoing study.

Thyroid hormones work in a feedback loop with your brain particularly your pituitary and hypothalamus in regulating the release of thyroid hormone. Your pituitary makes TRH (thyroid releasing hormone), and your hypothalamus makes TSH. If everything is working properly, you will make what you need and youll have the proper amounts of T3 and T4. Those two hormones T3 and T4 are what control the metabolism of every cell in your body. But their delicate balance can be disrupted by nutritional imbalances, toxins, allergens, infections, and stress. If your T3 is inadequate, either by insufficient production or not converting properly from T4, your whole system suffers. You see, T3 is critically important because it tells the nucleus of your cells to send messages to your DNA to crank up your metabolism by burning fat. That is why T3 lowers cholesterol levels, regrows hair, and helps keep you lean.

\section{How to know if you are Hypothyroid}

Identifying hypothyroidism and its cause is tricky business. Many of the symptoms overlap with other disorders, and many are vague. Physicians often miss a thyroid problem since they rely on just a few traditional tests, so other clues to the problem go undetected. But you can provide the missing clues! The more vigilant you can be in assessing your own symptoms and risk factors and presenting the complete picture to your physician in an organized way, the easier it will be for your physician to help you. Sometimes people with hypothyroidism have significant fatigue or sluggishness, especially in the morning. You may have hoarseness for no apparent reason. Often hypothyroid people are slow to warm up, even in a sauna, and do not sweat with mild exercise. Low mood and depression are common. Sluggish bowels and constipation are major clues, especially if you already get adequate water and fiber.

\section{Are the upper outer third of your eyebrows thin or missing?}

This is sometimes an indication of low thyroid. Chronic recurrent infections are also seen because thyroid function is important for your immune system. Another telltale sign of hypothyroidism is a low basal body temperature (BBT), less than 97.6degrees F4 averaged over a minimum of 3 days. It is best to obtain a BBT thermometer to assess this.

\section{How about your family history? Do you have close relatives with thyroid issues?}

Some of the family history that suggests you could have a higher risk for hypothyroidism includes:
i. High or low thyroid function
ii. Goiter
iii. Prematurely gray hair
iv. Left-handedness Diabetes

v. Autoimmune diseases (rheumatoid arthritis, lupus, sarcoidosis, Sjogren, etc.)

vi. Crohns disease or ulcerative colitis

vii. Multiple sclerosis (MS)

viii. Elevated cholesterol levels

It might be useful to take an online thyroid assessment quiz, as a way to get started. Mary Shomon has a good one, found here. Some of the classic symptoms are mentioned above, but there are many more too many to list here. If you suspect you might be hypothyroid, you should see a healthcare provider who can evaluate this, including ordering the basic lab tests for thyroid function.

\section{Laboratory testing}

Even though lab tests are not the end-all, be-all for diagnosing a thyroid problem, they are a valuable part of the overall diagnostic process. The key is to look at the whole picture. New studies suggest a very high incidence of borderline hypothyroidism in Westerners. Many cases are subclinical and even sub laboratory, not showing up at all in standard laboratory measurements. Coexistent subclinical hypothyroidism often triggers or worsens other chronic diseases, such as the autoimmune diseases, so the thyroid should be addressed with any chronic disease. Many physicians will order only one test a TSH level. This is a grossly inadequate and relatively meaningless test by itself, as well as a waste of your money. It would be like saying you know your water is pure because it tastes fine.

I recommend the following panel of laboratory tests if you want to get the best picture of what your thyroid is doing: TSH the high-sensitivity version. This is the BEST test. But beware most all of the normal ranges are simply dead wrong. The ideal level for $\mathrm{TSH}$ is between 1 and $1.5 \mathrm{mIU} / \mathrm{L}$ (milli-international units per liter) Free T4 and Free T3. The normal level of free T4 is between 0.9 and $1.8 \mathrm{ng} / \mathrm{dl}$ (nanograms per deciliter). T3 should be between 240 and $450 \mathrm{pg} / \mathrm{dl}$ (picograms per deciliter). Thyroid antibodies, including thyroid peroxidase antibodies and anti-thyroglobulin antibodies. This measure helps determine if your body is attacking your thyroid, overreacting to its own tissues (i.e., autoimmune reactions). Physicians nearly always leave this test out. For more difficult cases TRH (thyroid releasing hormone) can be measured using the TRH stimulation test. TRH helps identify hypothyroidism that caused by inadequacy of the pituitary gland. Other tests that might be indicated for more complex cases are a thyroid scan, fine-needle aspiration, and thyroid ultrasound. But these are specialized tests that your physician will use only in a small number of cases, in special situations. Even if all your lab tests are normal, if you have multiple thyroid symptoms, you still could have subclinical hypothyroidism. Keeping Your Thyroid Healthy in a Toxic World. Now that you have some understanding of the importance of your thyroid and how it works, let's take a look at the factors that can readily cause problems with your thyroid gland.

\section{Diet}

Your lifestyle choices dictate, to a great degree, how well your thyroid will function. If you follow my plan to eat for your nutritional type, 5 and my nutritional plan your metabolism will be more efficient, and your thyroid will have an easier time keeping everything in check. Eating for your type will normalize your blood sugar and lipid levels and enhance your immune system, so that your thyroid will have fewer 
obstacles to overcome. Eliminate junk food, processed food, artificial sweeteners, trans fats, and anything with chemical ingredients. Eat whole, unprocessed foods, and choose as many organics as possible.

\section{Gluten and other food sensitivities}

Gluten and food sensitivities are among the most common causes of thyroid dysfunction because they cause inflammation. Gluten causes autoimmune responses in many people and can be responsible for Hashimoto thyroiditis, a common autoimmune thyroid condition. Approximately 30percent of the people with Hashimoto thyroiditis have an autoimmune reaction to gluten, and it usually goes unrecognized.
I recommend using sea kelp with the Performance Pack metabolic to balance Thyroid Function.

\section{Acknowledgements}

None.

\section{Conflict of interest}

The author declares no conflict of interest. 